\title{
Sorafenib enhances mast cells maturation and degranulation in human skin
}

Yukari Mizukami, Koji Sugawara, Yukimi Kira, Daisuke Tsuruta Department of Dermatology, Osaka City University Graduate School of Medicine

\section{Introduction}

Sorafenib (Nexavar $\left.{ }^{\circledast}\right)$ is a multi-kinase inhibitor for treating hepatocellular and renal cell carcinomas by targeting various types of receptors and signaling molecules, including vascular endothelial growth factor receptors, platelet-derived growth factor receptor, and Raf-1.1)

Sorafenib is regarded as one of the most powerful options for treating these advanced cancers. ${ }^{2)}$

Sorafenib has been reported to cause diverse cutaneous reactions including itch. ${ }^{3)}$ However, the mechanism has not been well-investigated.

\section{Hypothesis}

Mast cells (MCs) could be related the mechanism of sorafenib-induced cutaneous adverse reactions because MCs are reported to be associated with various types of skin diseases including drug eruption and pruritus. ${ }^{4)}$

\section{Method}

To investigate the mechanism of sorafenib-induced adverse reactions, we focus on MCs by using human skin organ culture system ${ }^{5)}$ and skin samples from patients with drug eruption by sorafenib.

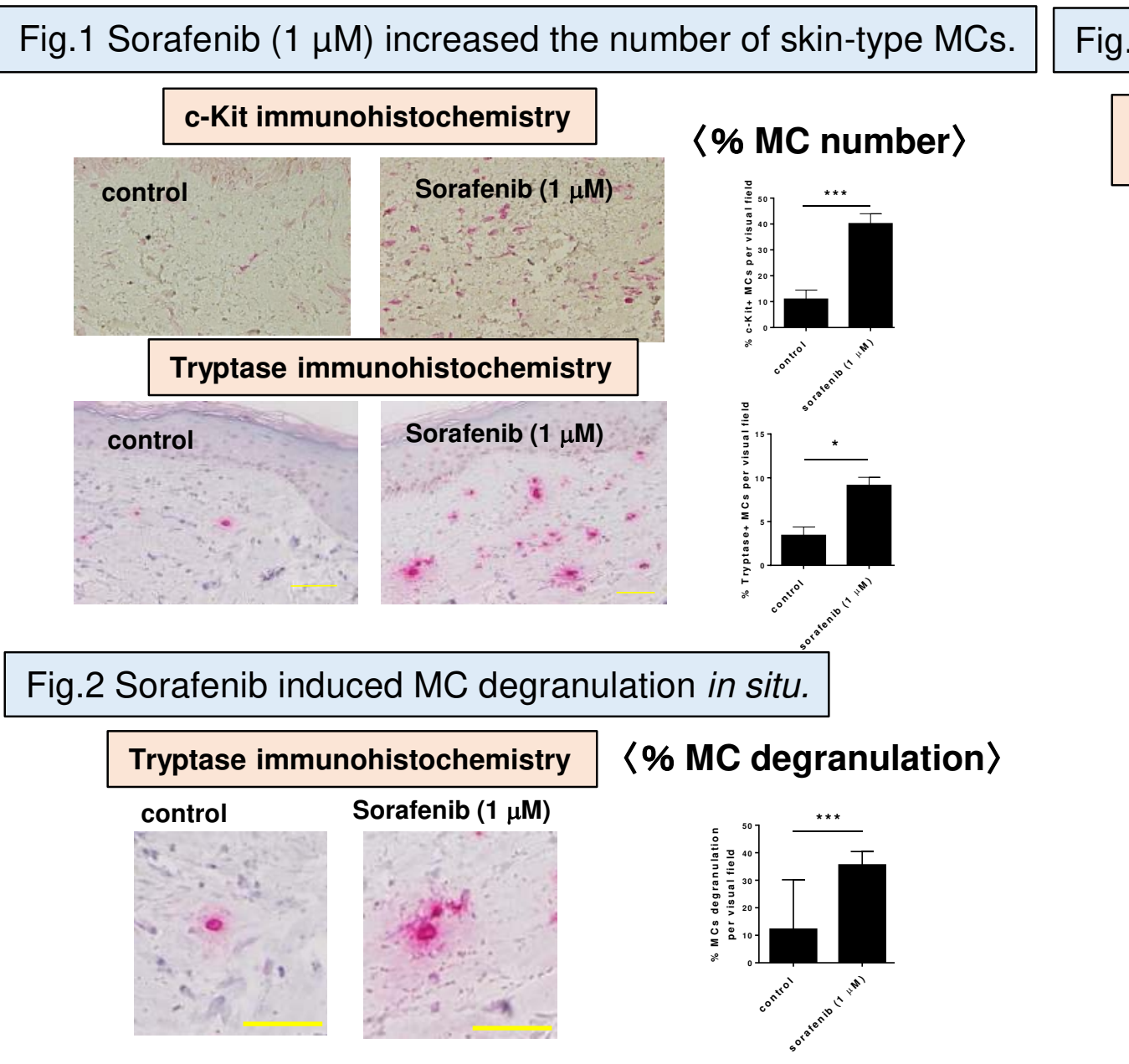

Sorafenib increased MC number and degranulation in situ.
Fig.3 Sorafenib may stimulate skin-type MC maturation in situ.

\begin{tabular}{c|}
\hline Tryptase /Ki-67 \\
double immunofluorescence \\
\hline
\end{tabular}

Tryptase /TUNEL
double immunofluorescence
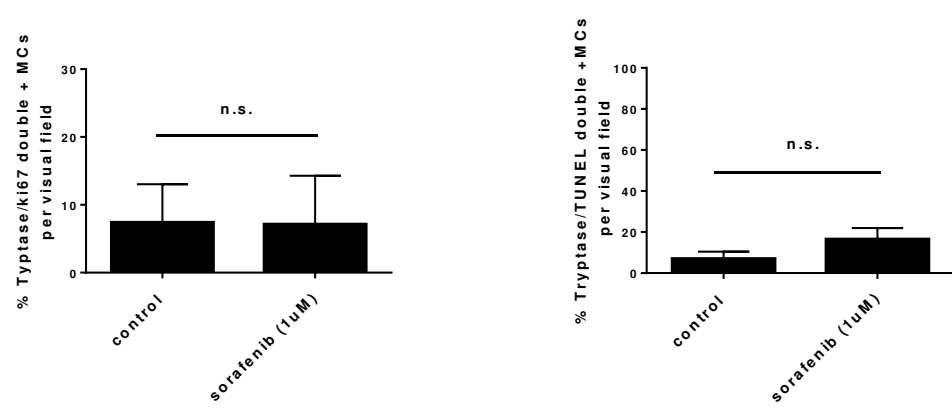

- Sorafenib did not affect MC proliferation and apoptosis.

- MC number in situ is controlled by their proliferation, apoptosis, and maturation.

This suggests that sorafenib stimulated MC maturation from resident precursor cells.
Fig.4 Sorafenib increased stem cell factor (SCF) expression.

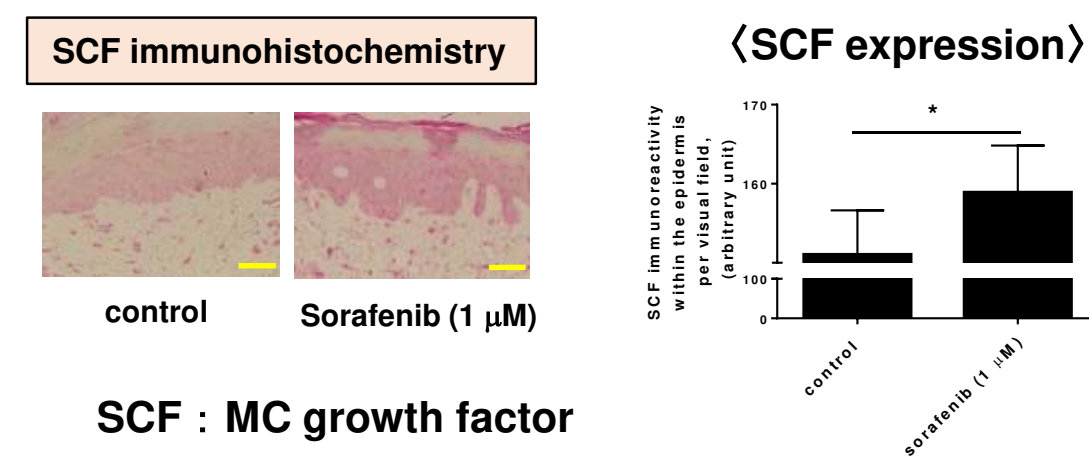

Fig.5. The co-administration of SCF neutralizing antibody diminished the increase in $\mathrm{MC}$ number by sorafenib.

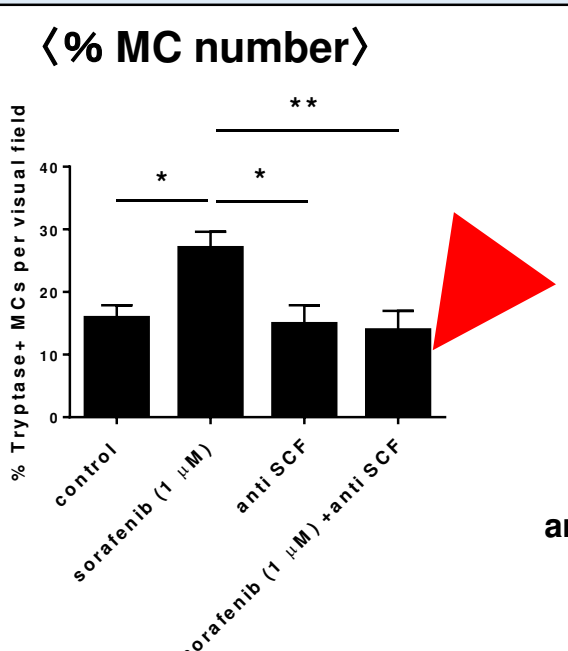

anti-SCF: stem cell factor neutralizing antibody
Fig.6 Compensatory upregulation of PI3K signaling was induced by the inhibition of MAPK signaling by sorafenib.

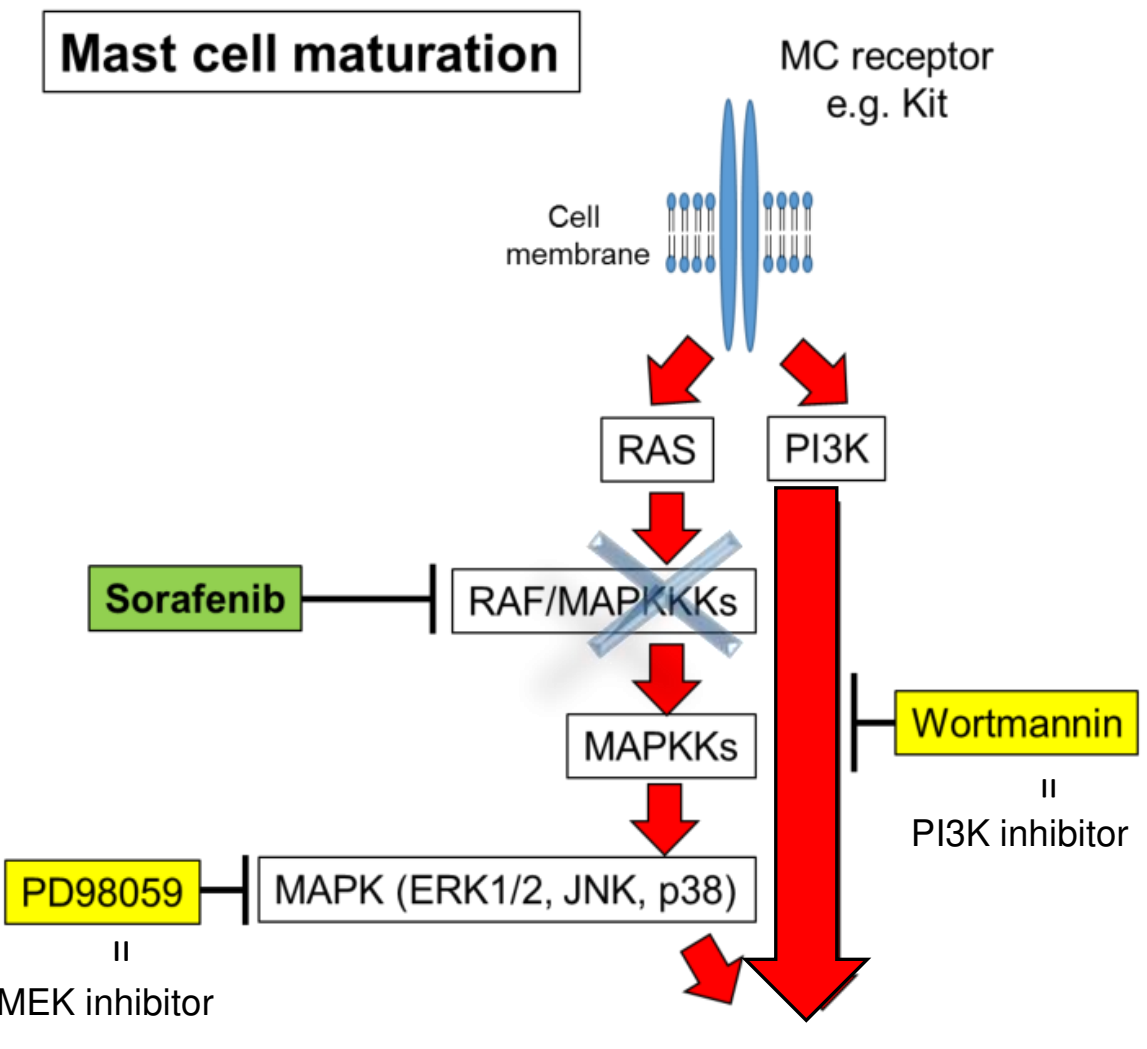

Mast cell maturation / growth / survival $\langle \%$ MC number〉

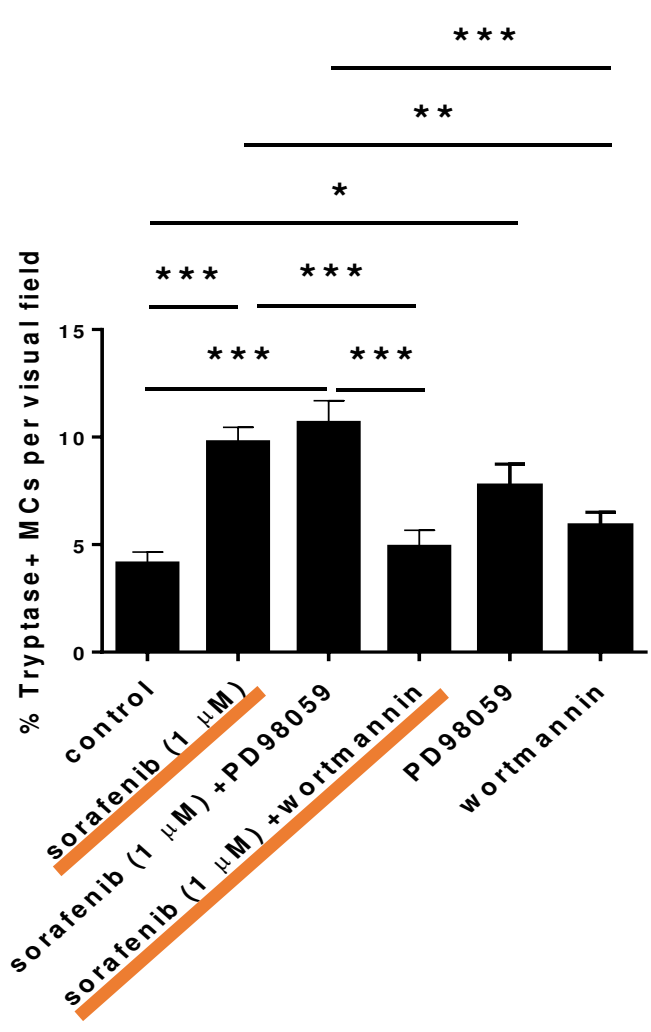

- PI3K inhibitor, wortmannin abrogated the increased number of MCs by sorafenib.

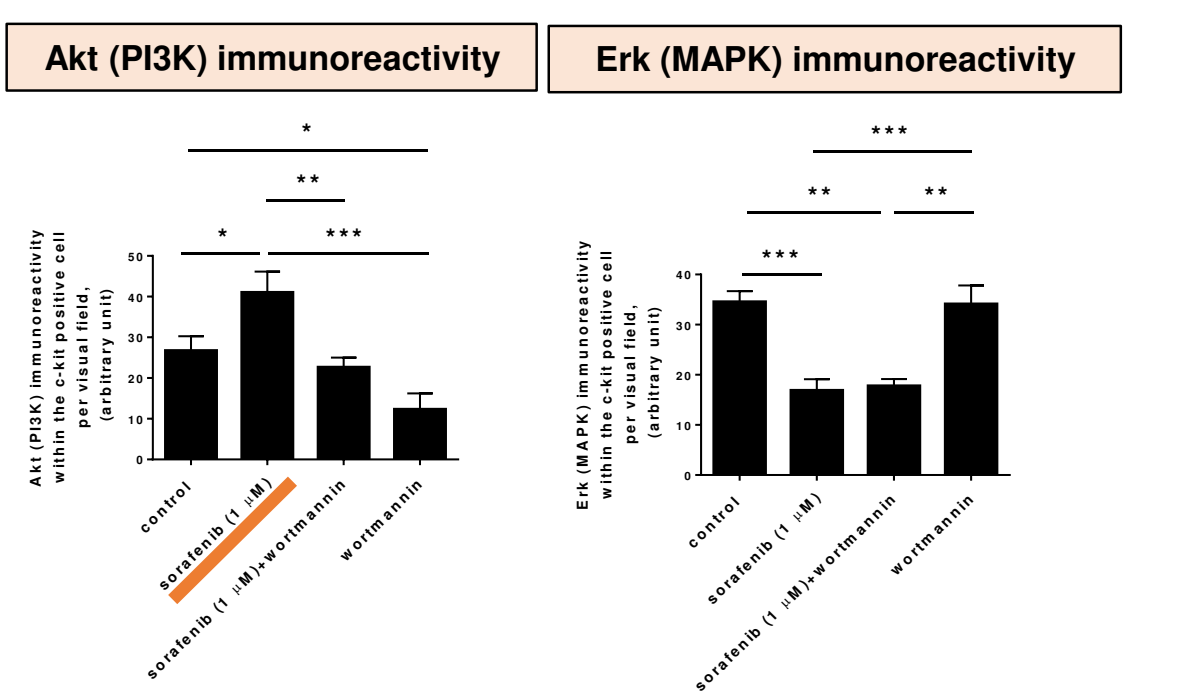

-Sorafenib significantly increased Akt (PI3K) immunoreactivity within the c-Kit+ MCs.

- The increased MCs induced by sorafenib may be controlled via the PI3K signaling pathway.

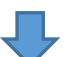

This suggests that the compensatory upregulation of PI3K-signaling was induced by the inhibition of MAP kinase signaling by sorafenib.
Fig.7 Sorafenib increased the number of tryptase MCs and degranulation and SCF in the skin lesions of patients treated within three weeks of sorafenib.

\begin{tabular}{|c|c|c|c|c|c|c|}
\hline Age & Sex & $\begin{array}{c}\text { Disease } \\
\text { treated } \\
\text { with Sorafenib }\end{array}$ & $\begin{array}{c}\text { Site of } \\
\text { skin biopsy }\end{array}$ & $\begin{array}{c}\text { Period from } \\
\text { administration of } \\
\text { Sorafenib } \\
\text { to skin biopsy }\end{array}$ & Types of drug eruption & $\begin{array}{c}\text { Patient's skin } \\
\text { sensation }\end{array}$ \\
\hline 61 & M & RCC & knee & 7 days & EMtype & itch \\
\hline 51 & M & RCC & hand & 7 days & hand-foot reaction & painful \\
\hline 22 & F & HCC & upper arm & 10 days & EMtype, Alopecia & itch \\
\hline 63 & F & RCC & forearm & 11 days & EMtype & itch \\
\hline 68 & F & HCC & chest & 14 days & EMtype & painful \\
\hline 85 & F & RCC & upper arm & 14 days & EMtype & itch \\
\hline 65 & F & TC & upper arm & 21 days & Maculopapular type & itch \\
\hline 56 & M & HCC & femur & 47 days & EMtype, Pustular drug eruption & itch \\
\hline RCC: renal cell carcinoma, HCC: hepatocellular carcinoma, TC: thyroid cancer, EM: erythema multiforme &
\end{tabular}

When we investigated MCs biology within the skin samples from the patients with sorafenib induced drue eruptions,

the number of degranulated and total MCs and SCF expression was significantly increased.

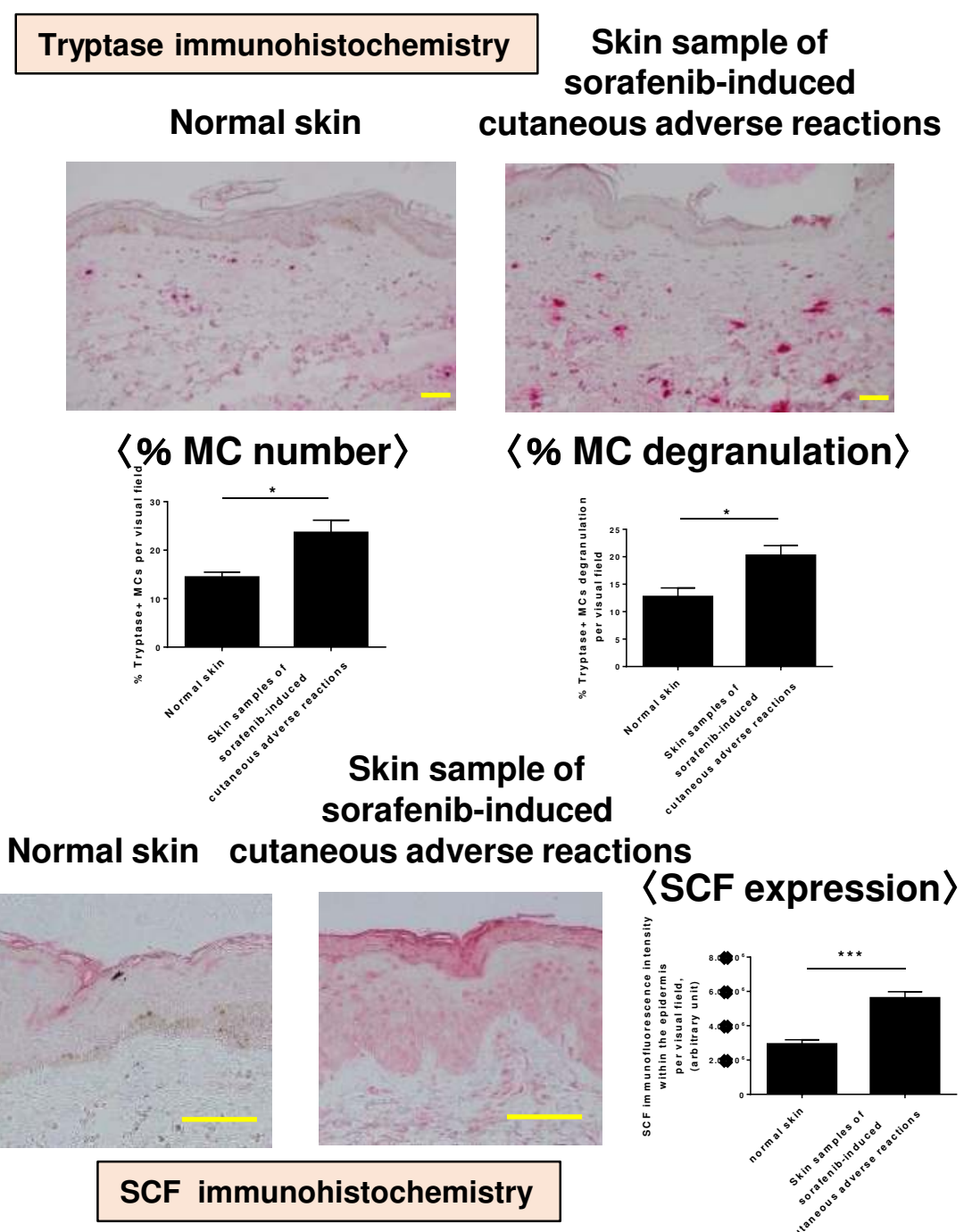

1. Sorafenib increased mast cell number and stimulates mast cell maturation and induces degranulation.

2. Sorafenib also increases SCF expression.

3. The effect of sorafenib on mast cell maturation is mediated via PI3K signaling.

4. PI3K mediated signaling or SCF could be a new target for sorafenib-induced itch.

Sorafenib induces itch via activation of degranulation and maturation of skin-type MCs.

This activation is related to PI3K signaling and SCF production, which could be new targets for treating sorafenib-induced cutaneous reaction including itch. 